\title{
The Numerical Simulation of flow pattern in Horizontal Mutation Pipe
}

\author{
Shupeng Cai, Binwen Luo, Yaode Qiu, Peng Gao
}

School of Mechanical Engineering, Hunan University of Technology, Zhuzhou 412007, China

Keywords: Mutation pipe; expansion ratio, inclination angles of mutation pipe; re-attachment length; local resistance coefficient.

\begin{abstract}
In order to explore the flow characteristics in Horizontal Mutation Pipe, an SIMPLEC algorithm was used to simulate the flow field in horizontal mutation pipe by using the computational fluid dynamics software of Fluent. The variation of the length of entrance zone and the local resistance coefficient can be optimized by analyzing regulation of their effect on entrance velocity, the ratio of tube and the inclination angles. The results indicate that the re-attachment length relate to expansion ratio and inclination angles; the local loss coefficient increases with the increment of expansion ratio whereas decreases with the decrement of the inclination angles.
\end{abstract}

\section{Introduction}

The fluid in sudden expansion pipe has inertia, the mainstream gradually growing large along the pipeline axis if it encounters variable cross-section. The fluid formed a vortex between wall and the mainstream, the vortex driven by the mainstream, which in turn resulted in the energy losses of the mainstream. At the same time, the vortex, driven by the mainstream, was moving to the downstream. This caused greater turbulence intensity and local head loss within limits ${ }^{[1]}$. When cross-section changes suddenly, the flow field appears a series of complex dynamic phenomena such as inverse flow, separation, re-attachment, and shearing ${ }^{[2-3]}$. Due to the mutation tube was used widely in industrial field, it has important implications for the numerical simulation of the flow field in horizontal mutation pipe.

As one of the basic pipe elements, the hydraulic characteristics study of the mutation pipe played a key role in network optimization. Some research of mutation pipe flow had been published. For example: Li Donghao et al. ${ }^{[4]}$ measured the local head loss for three different operating conditions. Based on the experiment result, the influences of the local resistance coefficient which caused by flow rate, pipe diameter, and water temperate had been analyzed. The paper concluded that it was the linear relation between local resistance and logarithms of inlet velocity. Sun $\operatorname{Lin}^{[5]}$ had carried through numerical simulation and experimental study of four different expansion ratio and three different shrinkage ratio models. The result showed that almost all local coefficient of mutation pipe are associated with inlet velocity. The local coefficient of expansion pipe increased with increasing expansion ratio. Currently, the influence of inclination angle on the local resistance coefficient and the factors influencing the re-attachment length in mutation pipe was few in writing. But the re-attachment length was important basis for judging no back flow occur in near wall area and the local resistance coefficient was important theoretical parameter in engineering. Therefore, the mutation pipe flow field with the different inlet velocity, expansion ratio and inclination angle was simulation calculated by using fluent software. The paper explores the factors influencing the re-attachment length and the impact of the local resistance coefficient on the inclination angle in mutation pipe.

\section{The calculation model and grid}

\subsection{The calculation model}

Under the condition of low inlet velocity, pipe flow could be described as an axis-symmetric flow due to having strong two-dimensional flow characteristics in mutation pipe ${ }^{[6]}$. To reduce the amount of calculation, the paper has simplified mutation pipe flow to axis-symmetric flow. The mutation tube 
structure was shown in fig.1, the small tube diameter $D_{1}$ was $10 \mathrm{~mm}$, three expansion ratio $E=D_{2} / D_{1}$ was $1.5,2.0$, and 2.5 respectively. The small tube length $L_{1}=150 D_{1}, L_{2}=50 D_{2}$. The inclination angle $\alpha$ has the ranged of $10^{\circ} \sim 90^{\circ}$.

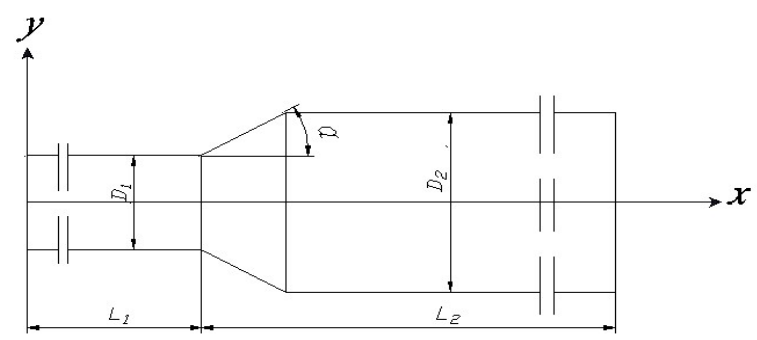

Fig. 1 The schematic diagram of horizontal mutation pipe

\subsection{Grid division}

The two-dimensional model for mutation pipe was set up by GAMBIT software. The quadrilateral meshes are generated for the whole structure model by using the separated region template method and refined grid near the boundary. Fig. 2 shows the mesh pictures for inclination angles to $30^{\circ}$.

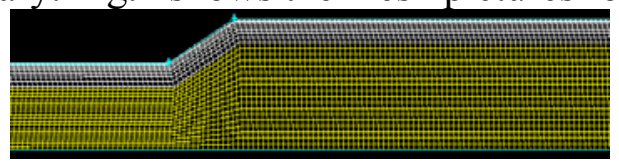

Fig. 2 Mesh model of horizontal mutation pipe

\section{3 boundary conditions and algorithm}

The paper adopts velocity inlet and pressure outlet as boundary condition. The inlet velocity was $0.1 \sim 3 \mathrm{~m} / \mathrm{s}$ in the range of $1000 \sim 30000$ for Reynolds number. The pressure of outlet was equal to standard atmospheric pressure. The boundary conditions near the solid walls had been analyzed by using the method of standard wall function.

\section{Simulation results and analysis}

\subsection{The influence of expansion ratio on re-attachment length}

The model of mutation pipe had been numerically simulated when the expansion ratio $E$ were 1.5, 2.0, 2.5 and the inlet velocity was $1 \mathrm{~m} / \mathrm{s}$. The paper draws the fluid streamlines by Tec-plot software, as shown in Fig.3.

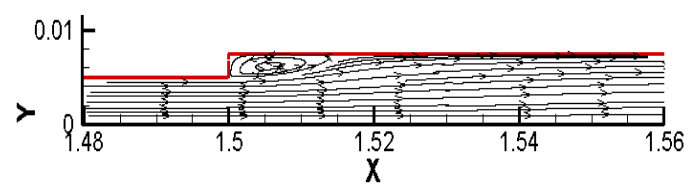

a) $\mathrm{E}=1.5$

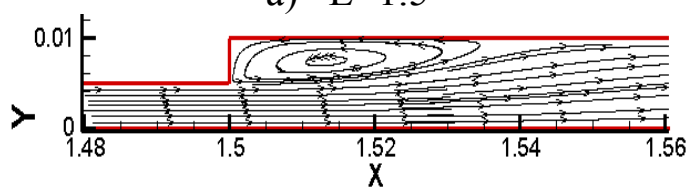

b) $\mathrm{E}=2.0$

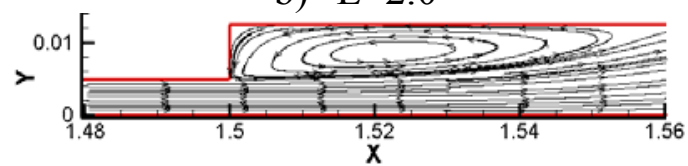

c) $\mathrm{E}=2.5$

Fig. 3 the streamline figures with different the ratio of tube

As can be seen in Fig.3, the size of vortex increased with the increasing expansion ratio when the inlet velocity remains unchanged. The vortex condense mainstream, it makes the velocity redistribute near wall region. So vortex size increasing resulted in the back of the velocity gradient position. The 
rotation energy loss of vortex was come from the mainstream beam by momentum exchange and fluid viscosity. Therefore, the greater size of vortex, the more energy consumed, and then the more energy was absorbed from the mainstream. So the increasing expansion ratio will lead to the increase of energy loss.

Fig.4 shows the variation laws of the re-attachment length with inlet velocity at different expansion ratio. The re-attachment point was defined by derivation the radial velocity curve near wall region. $L$ is the re-attachment length from the enlargement section to the re-attachment point. In fig.4, the ratio $L / H$ is shown the change of re-attachment length $L, H=\left(D_{2}-D_{1}\right) / 2$.

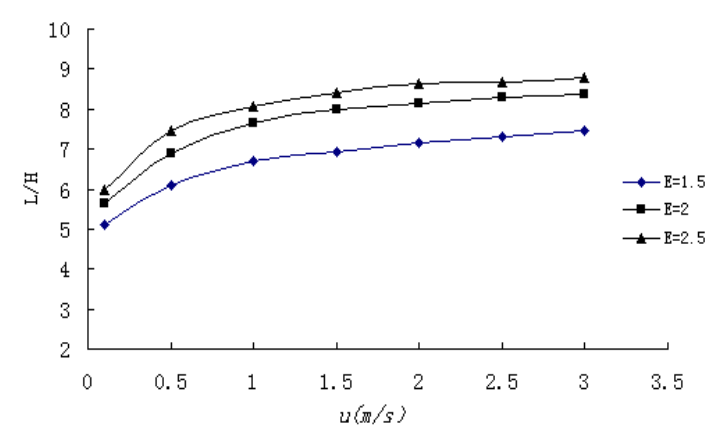

Fig.4 The relationship between re-attachment length and the entrance velocity under expansion ratio

As can be seen from the fig.4, the re-attachment length increased with the increasing of inlet velocity and finally reached a constant. It shows that the recirculation zone length near the sudden-changed cross-section increased with the increasing inlet velocity and finally reached a constant. The re-attachment length increased with the increasing of expansion ratio when the inlet velocity was a constant.

\subsection{The influence of inclination angle on re-attachment length}

The model of mutation pipe had been simulated when the expansion ratio and inlet velocity were $1.5,1 \mathrm{~m} / \mathrm{s}$ respectively and inclination angles range within $10^{\circ} \sim 90^{\circ}$, as shown in fig.5.
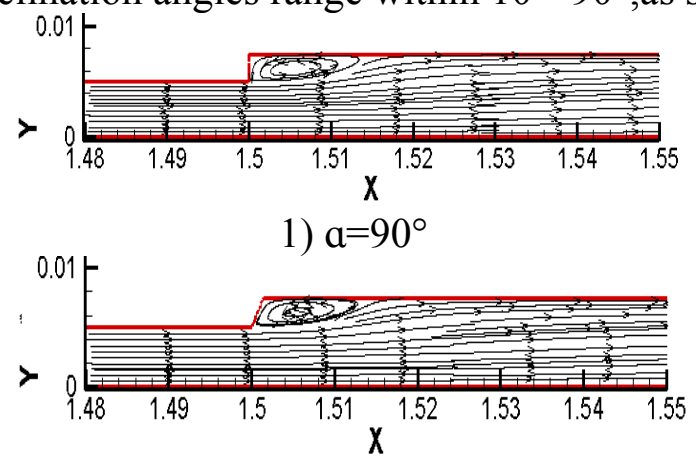

2) $a=60^{\circ}$

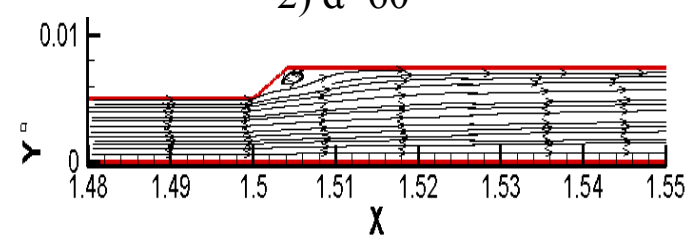

3) $a=30^{\circ}$

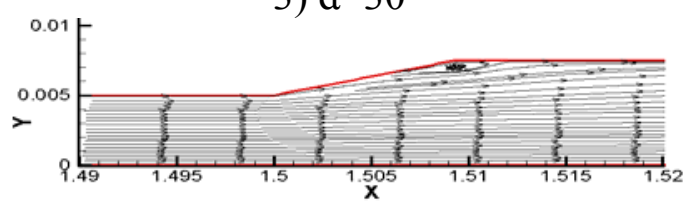

4) $a=15^{\circ}$ 


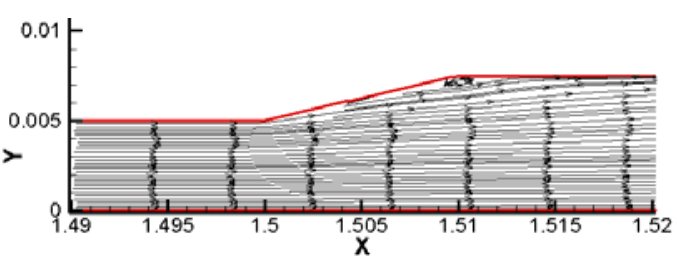

5) $a=14^{\circ}$

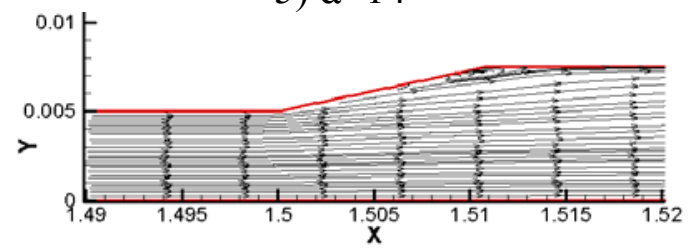

6) $a=13^{\circ}$

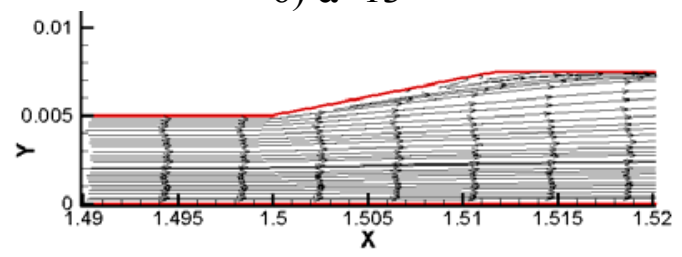

7) $a=12^{\circ}$

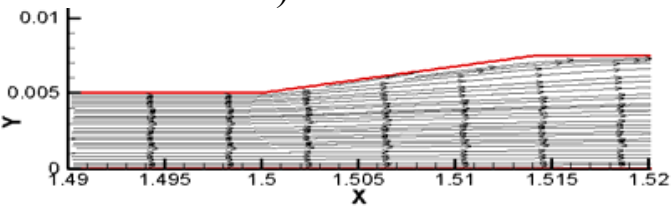

10) $a=10^{\circ}$

Fig. 5 The streamline figures with different inclination angles

With the comparison of the fluid streamlines, the size of vortex decreased with the decreasing of inclination angles. It's hard to see the presence of the vortex in the fluid streamlines figures when the inclination angle was equal to $13^{\circ}$. As can be seen in the fig. 5 , the smaller vortex was generated due to the closer wall which makes the front section of vortex squeezed among the wall and the mainstream line. The large vortex was hardly produced mainly by the smaller space zone between the mainstream line and the boundary as a result of the smaller inclination angle.

Fig. 6 show the change law of re-attachment length with inclination angles when inlet velocity was $1 \mathrm{~m} / \mathrm{s}$.

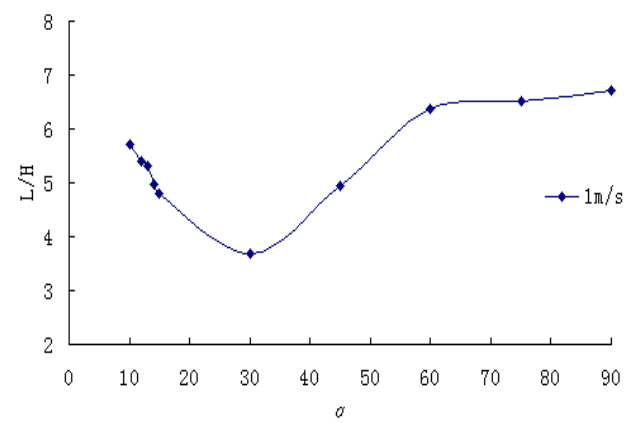

Fig. 6 The relationship between re-attachment length and inclination angles

As can be seen from fig.6, the minimum and maximum point of re-attachment length emerged when inclination angles were $30^{\circ}$ and $90^{\circ}$, respectively. Therefore, with increase of inclination angle, the re-attachment length decrease first and then increase. When inclination angle was in the range of $10^{\circ} \sim 30^{\circ}$, since the too small inclination angle cause the later vortex position, it makes the re-attachment length longer. So when the inclination angles range within $10^{\circ} \sim 30^{\circ}$, factor affecting re-attachment length was inclination angle of mutation pipe. When inclination angle range within $30^{\circ} \sim 60^{\circ}$, the vortex position move forward with inclination angle increases, but it reduces the 
suppression capacity of mutation pipe and increases the size of vortex. So when the inclination angles in range of $30^{\circ} \sim 60^{\circ}$, factor affecting re-attachment length was inclination angle and the vortex. When inclination angle range within $60^{\circ} \sim 90^{\circ}$, the size of the vortex makes little affected by the inclination angle of mutation pipe. So when the inclination angles range within $30^{\circ} \sim 60^{\circ}$, the major factor affecting re-attachment length was the vortex.

\subsection{The influence of expansion ratio on the local resistance coefficient}

In engineering fluid mechanics, the formula of local head loss was given by formula (1)

$$
h=\zeta \frac{u^{2}}{2 g}
$$

In formula (1): $\zeta$ was the local resistance coefficient; $u$ was the average flow rate of the smaller tube; $g$ was the gravitational acceleration. The formula of local resistance coefficient was given by formula (2)

$$
\zeta=\left(1-\frac{A_{1}}{A_{2}}\right)^{2}
$$

In formula (2): $A_{1}$ and $A_{2}$ were the cross-sectional abrupt change of mutation pipe front and back respectively.

The local resistance coefficient of expansion ratio $E=1.5,2.0,2.5$ are $0.3086,0.5625$ and 0.7056 respectively by formula (2)

Fig.7 shows the variation laws of local resistance coefficient with inlet velocity by simulation calculation under the different expansion ratio.

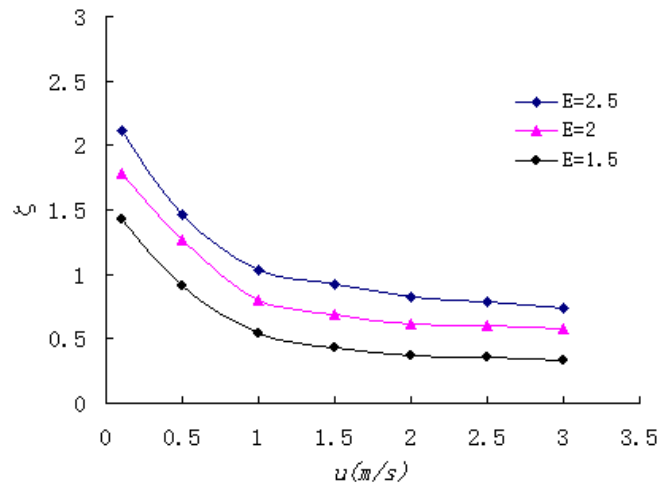

Fig. 7 The relationship local resistance coefficient and inlet velocity under different expansion ratio

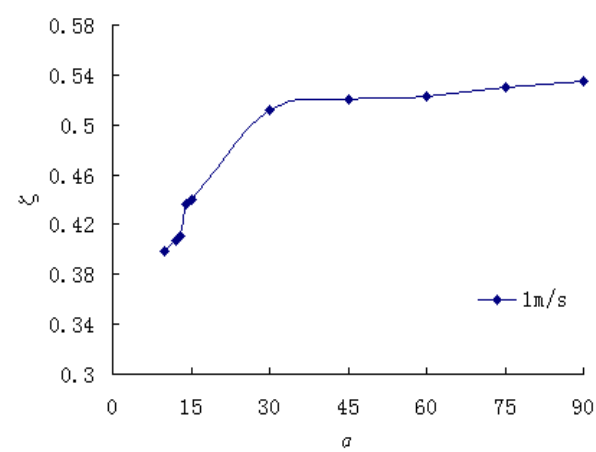

Fig. 8 The relationship between local resistance coefficient and inclination angles

Form the figure7, the local resistance coefficient decreased with the increasing of inlet velocity under the smaller inlet velocity, and later on it approach to constant. The resistance coefficient relates mostly to the expansion ratio when the inlet velocity over a certain constant. The local resistance coefficient increased with the increasing of expansion ratio under the same inlet velocity. The above two trends were presented mainly by the larger vortex as the increasing of inlet velocity and expansion ratio. The rotation energy loss of vortex was come from the mainstream line by fluid adhesiveness. The local resistance coefficient is all about the sudden-changed cross-sectional area in 
engineering fluid mechanics. But in fig.7, the local resistance coefficient has changed dramatically in mutation pipe with increasing of inlet velocity under the smaller Reynolds number, because while the laminar flow passes the sudden-changed cross-section and then always maintain laminar flow, the more intense relative motions between the laminar flows was produced. It caused the more energy loss in the regionally.

\subsection{The impact of inclination angle on local resistance coefficient.}

The model of mutation pipe had been simulated when the expansion ratio was 1.5; the inlet velocity was $1 \mathrm{~m} / \mathrm{s}$, the inclination angles range within $10^{\circ} \sim 90^{\circ}$. Fig. 8 shows the variation laws of local resistance coefficient with inclination angle by simulation calculation when the expansion ratio is 1.5 .

As shown in fig. 8 , the local resistance coefficient decreased with the decreasing of inclination angle. The head loss of the mainstream beam decreased due to the smaller inclination angle which makes the stronger suppression vortex capacity of the mutation pipe. When inclination angle range within 10 30 degree, the local resistance coefficient obviously change with decreasing inclination angle As a result, when inclination angle was less than 30 degree, the capability of mutation pipe restraining the loss-energy resulting from flow deformation was expanded. So, the inclination angle of best mutation pipe should be less than $30^{\circ}$, preferably as small as possible.

\section{Conclusions}

The flow characteristics in horizontal mutation pipe had been studied with different inlet velocity, expansion ratio and inclination angle by Fluent software. The paper analyzed the influence of each variable to re-attachment length and local resistance coefficient. The simulation results show that:

1) The re-attachment length depends on inclination angles of mutation pipe and vortex in flow field.

2) The local resistance coefficient of mutation pipe decreases with increasing flow rate and tends to a constant.

3) The local resistance coefficient of mutation pipe was not only depends on the sudden enlargement ratio, but it was associated with the expansion ratio. The local resistance coefficient of mutation pipe increased with increasing sudden enlargement ratio, and decreases with decreasing expansion ratio.

\section{Acknowledgements}

This work is being supported by the National Natural Science Foundation of China through grant No. 51476051.

\section{References}

[1] Zhou Zaidong,Wei Changzhu,Sun Mingyan,et al. Sudden Expansion Pipe Flow Pattern of the Numerical Simulation[J]. Science Technology and Engineering,2012,12(30):185-187.

[2] Yin Zegao,Zhang Tuqiao,Huang Yadong,et al. Numerical Simulation of Serial Sudden Expansions Pipe Flow[J]. fluid Machinery,2005,33(8):24-27.

[3] Wang Xiaohua,Ju Shuohua,Zhu Wenfang. Numerical Simulation of Flow at Sudden Expansion[J].LowTemperat.Architect.Technology,2003(1):59-60.

[4] Li Donghao,Wang Wene,Ge Maosheng,et al. Study on Local Drag Parameter of Subcontract Tube[J]. Journal of Water Resources and Architectural Engineering, 2011,9(4):22-24.

[5] Sun Lin. Experimental Study and Flow Field Numerical Simulation on Loss of Pipe with Sudden Expansion or Contraction[D]. Harbin:Northeast Agricultural University,2010.

[6] Gao Dianrong,Zhang Qisheng. Finite Element Simulation of Transient Flow in a Planar Sudden Expansion Channel[J]. Jouranl of Yanshan University,1999; 23(4):331-334 